\title{
Simulation and evaluation of WIMAX handover over homogeneous and heterogeneous networks
}

\author{
Anmar Hamid Hameed ${ }^{1,2,{ }^{*} \text {, Salama A. Mostafa }}{ }^{1,2}$, Mazin Abed Mohammed ${ }^{3,4}$ \\ ${ }^{1}$ College of Graduate Studies, Selangor, Malaysia \\ ${ }^{2}$ Universiti Tenaga Nasional, Selangor, Malaysia \\ ${ }^{3}$ Dept. of Planning and Follow up, Anbar, Iraq \\ ${ }^{4}$ University of Anbar, Anbar, Iraq \\ Email address: \\ anmar_oio@yahoo.com(A. H. Hameed),semnah@yahoo.com(S. A. Mostafa),mazin_top_86@yahoo.com(M. A. Mohammed)

\section{To cite this article:} \\ Anmar Hamid Hameed, Salama A. Mostafa, Mazin Abed Mohammed. Simulation and Evaluation of WIMAX Handover over \\ Homogeneous and Heterogeneous Networks. American Journal of Networks and Communications. Vol. 2, No. 3, 2013 , pp. 73-80. \\ doi: 10.11648/j.ajnc.20130203.14
}

\begin{abstract}
Different aspects of wireless networks are changing as a result of the continuous needs in terms of speed, data rates and quality of service. Such aspects are required to be adaptable within the same network or among networks of different technologies and service providers. Consequently, Worldwide Interoperability for Microwave Access (WiMAX) is one of the future generation networks $(4 \mathrm{G})$ that needs further study. However, a major consideration for WiMAX to achieve mobility is the handover mechanism that concerns with the mobile station movement within the range of network coverage from one base station to another similar homogeneous or different homogeneous network. In this paper, an intensive analysis to handover mechanism in WiMAX followed by comparisons to WiMAX performance with UMTS and WiFi networks is carried out. QualNet 4.5.1 simulator is used to simulate the comparison process homogeneously and heterogeneously. Performance metrics of Throughput, End-to-End (E-2-E) Delay and Jitter in the comparison during handover process $\mathrm{in} / \mathrm{between}$ the wireless networks are used. The simulation results are evaluated to identify the performance of the handover process over WiMAX-WiMAX, WiMAX-UMTS and WiMAX-WiFi with respect to the selected metrics. The environment of Wi-MAX-WiMAX has shown substantial enhancement of the system Throughput, reduction of E-2-E Delay and Jitter.
\end{abstract}

Keywords: Homogeneously and Heterogeneous Networks, WiMAX, WiFi, UMT, Handover Mechanism

\section{Introduction}

The world is moving to the age of velocity in every field especially the wireless networks field. To go along with it, it is needful to have faster facilities, more importantly in the wireless networks. The need is to provide mobile wireless with higher data rates, Quality of Service (QoS) and adaptability within the same network or among networks of different technologies and service providers. The users should be able to get its potential whatever they are using (PC, cell phone, electronic pad, etc. wise), and where ever they are sitting; at home, walking and even driving [1].

Worldwide Interoperability for Microwave Access (WiMAX) is one of the future generations (4G) promising networks to cover some of the consumers' needs. It is an emerging technology that is designed to deliver fixed, and more recently, mobile broadband connectivity [2]. The WiMAX trade name is used to group a number of wireless technologies that have emerged from the Institute of Electrical and Electronics Engineers (IEEE) 802.16 Wireless Metropolitan Area Network (MAN) standards [3]. The main standards introduce mobility and currently receive a great deal of interest in the telecoms world [2]. Figure 1 show environments that can be employed in WiMAX [1].

The IEEE community has defined the IEEE 802.16e amendment (i.e. mobile WiMAX) to support mobility. Mobile WiMAX of IEEE 802.16e defines wireless communication for mobiles, moving at speed of $125 \mathrm{KMPH}$ in the range of $2-6 \mathrm{GHz}$ (802.16e). IEEE 802.16e is implemented with Orthogonal Frequency-Division Multiple Access (OFDMA) as its physical layer scheme [4]. Full mobility technology is different from one network to another, and each technology has its own mobility characteristics [5]. To ensure high level of mobility, it is important for WiMAX to have an efficient handover mechanism [6]. 


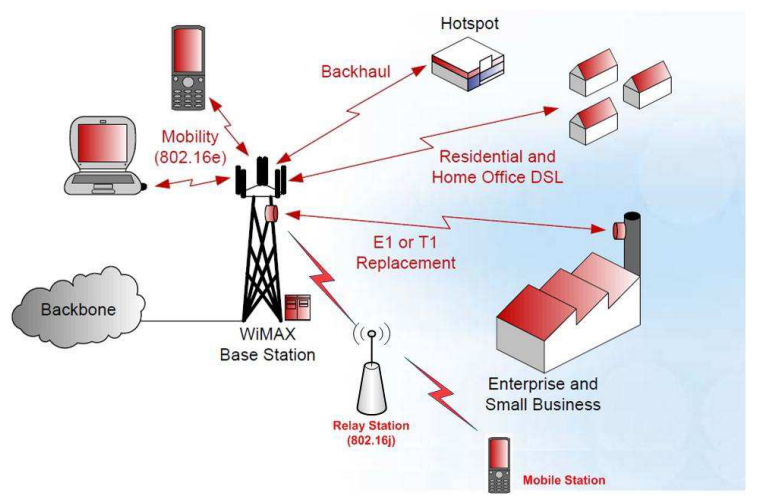

Figure 1. WiMAX possible environments

This paper investigates the WiMAX mobility capabilities and issues related to homogenous (between the same types of networks) and heterogeneous (between different types of networks) mobility. Specifically, the paper focuses on the handover performance of the WiMAX in comparison with similar WiMAX network or with other networks. For performance evaluation, Throughput, End-to-End (E-2-E) Delay and Jitter metrics are used as networks performance quality criteria to investigate the WiMAX mobility capabilities and efficiency of handover process in the comparison with other wireless technologies.

\section{Objectives}

Due to the importance and the complication of the mobile wireless networks, such as the WiMAX, UMTS and WiFi networks [7], an analysis study that aids in understanding the mobility problems, mobility insights from requirements, parameters and configurations in Mobile WiMAX network is presented in this research. The main objective focuses of the research are as follows:

1. To analysis mobile WiMAX network with regards to network topology acquisition, the base station and the main management messages that rules the mobility in WiMAX systems.

2. To evaluate mobile WiMAX network by performing an actual handover process to a new target base station.

3. To compare WiMAX performance with UMTS and WiFi wireless networks with respect to Throughput, E-2-E Delay and Jitter.

\section{Problem Analysis}

There are number of means to support mobility in communication devices such as roaming, portability, and covering mobility with a faster speed (vehicle speed) [9]. However, one of the important concerns of mobility concept for communication devices is how to motivate end users to use this technology, together with the emerging services for mobile devices presented by the service provider e.g. using packet data for streaming multimedia files [8].

As there are many wireless networks technologies, there is a need to gather and analyze information about these technologies to be filtered and collected in a trendy presentation [10]. Communication devices such as smart phones and laptops capabilities are getting more complex and emergent services that are supplied by the network provider like streaming audio/video through packet data are more desirable [6]. More so, there are different methods behind the cutting edge of the overall system performance for instance portability, full mobility and roaming. These methods often differ among various network technologies as the mobility characteristics of each [10].

For the reasons above, it is vital to present a comprehensive study about mobile WiMAX and awareness of its mobility capabilities especially in the motion state and comparing it with other types of technologies. Consequently on one hand, knowing how WiMAX supports intra-network mobility, and is it better than the other contemporary technologies? And on the other hand, the handover mechanism as it is considered as an important process included within mobile WiMAX technology is not intensively reviewed [11]. As a matter of fact, researchers elaborated a handover in general or simulated the handover homogeneously within one network $[7,12]$.

\section{Literature Review}

Worldwide Interoperability for Microwave Access (WiMAX) is a new wireless technology that can be used to build a wide coverage area networks with high Throughput [13], and high security [14] as shown in Figure 1. It is intended for Wireless Metropolitan Area Network (WMAN). WiMAX used to be the buzzword of wireless communication industry for the last six years. It is able to provide Broadband Wireless Access (BWA) up to 30 miles $(50 \mathrm{~km})$ for fixed stations, and 3-10 miles $(5-15 \mathrm{~km})$ for mobile stations [14]. IEEE authorized in 1999 a new working group known as 802.16. The group published its first standard, IEEE 802.16a, in January 2003 [15]. The standards of WiMAX are identified as 802.16-2004 (October 2004), 802.16e (December 2005), 802.16j (Jun 2009) and $802.16 \mathrm{~m}$ (still under development) [16]. Figure 2 shows the number of WiMAX deployments over time per frequency.

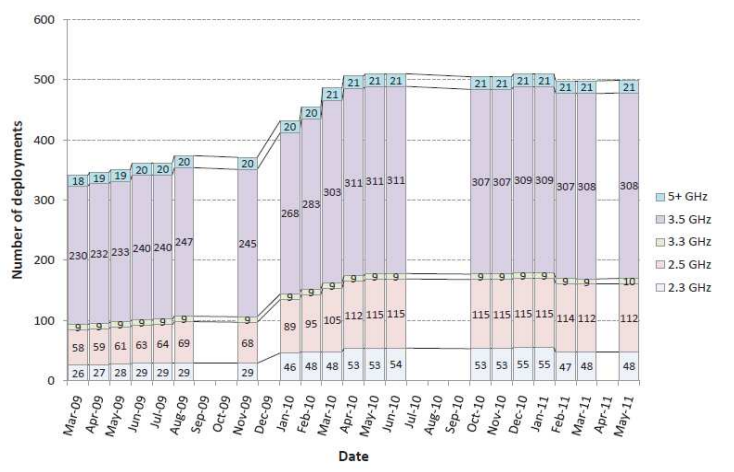

Figure 2. Cumulative number of WiMAX deployment per frequency [17] 
Daan P. et al. [17], have elaborated the different activities that occurred within the three important organizations: The 802.16 working group of the IEEE for technology development and standardization, the WiMAX forum for product certification and the ITU (International Telcomunication Union) for international recognition. They presented a comprehensive overview about the evolution of WiMAX in terms of standardization and certification. Furthermore, they highlighted the steps that have been considered in cooperating with the ITU to improve the international esteem of the technology. Finally, WiMAX trend analysis has also been discussed.

Ray et al. [18], proposed a fast and simple scheme for Mobile Station (MS)-controlled handover mechanism for mobile WiMAX environment. They suggested Received Signal Strength (RSS) and path-loss formula in estimating MS distance from any neighboring Base Stations (BS). The MS monitors the received signal strength that serves specific BS periodically. The MS-controlled approach simulation of Mobile WiMAX network outcomes show an observable reduction to handover latency accompanied by increase in network scalability.

\section{Methodology}

Since the WiMAX is a new technology, it is difficult to get a realistic comparison regarding a specific area of concern under specific circumstances within its architecture. Because of the lack practical experience with WiMAX and most of the experiments or simulations already done were focused on one specific area of concern. This research contributes comprehend study on mobile WiMAX network handover process performance.

The simulation of the handover process in mobile WiMAX is implemented using QualNet 4.5.1 simulator. Three performance matrices selected to be used in the experiment are: Throughput E-2-E delay, and Jitter within WiMAX environment. The obtained results are compared with the results obtained from the handover simulation process of WiMAX to WiFi network and WiMAX to UMTS network. Finally, the Uplink Channel Descriptor (UCD) and the Down Link Channel Descriptor (DCD) are simulated within two different time intervals during the handover process. In this section the research methodology is presented to illustrate how the research is carried out to meet its objectives.

\subsection{QualNet Simulator}

QualNet [19] is a state-of-the-art simulator for large, heterogeneous networks and the distributed applications that execute on such networks. QualNet [20] is simulating software that can be run on several platforms like Windows, Linux, O SX, and Solaris and it is capable of simulating wireless networks such as WiMAX [21]. QualNet has been used to simulate high-fidelity models of wireless networks with as many as 50,000 mobile nodes [22]. It uses architecture analogues of the TCP/IP network protocol stack which is a layered architecture.

The technology used in QualNet consists of 5 layers (top to bottom) as shown in Figure 3. The layers that are adjacent in the protocol stack communicate using well defined protocol. In general, the communication only occurs between adjacent layers (possible to be altered by the developers) [23].

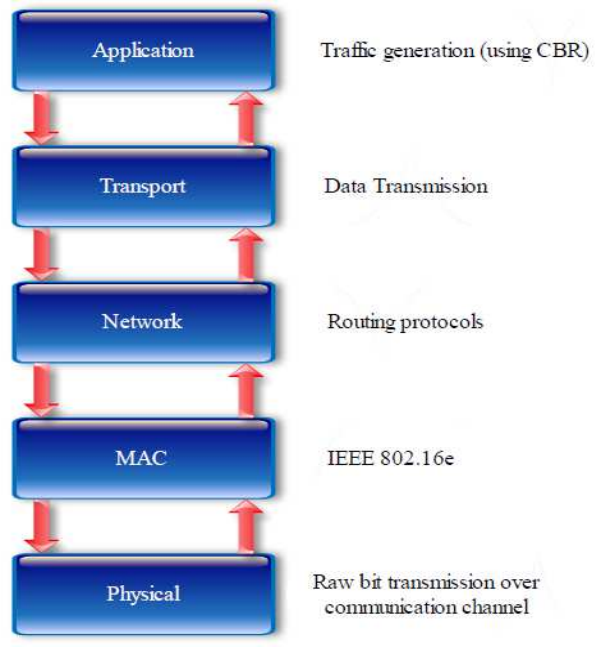

Figure 3. QualNet protocol stack

Figure 3 shows the QualNet layer model in which Constant Bit Rate (CBR) application and IEEE 802.16e $\mathrm{MAC}$ are used in the simulation. CBR application is generally used to simulate multimedia traffic (time critical traffic types). It can be configured to simulate a large number of real network applications by mimicking their traffic pattern and by filling traffic at a constant rate into the network. It can be accurately simulated by appropriately configuring the CBR application in QualNet. The simulated traffic sources CBR is generated using 4 mobile nodes performing handover process homogenously and heterogeneously with two time intervals, 5 and 10 seconds (UCD, DCD).

\subsection{Performance Metrics}

Performance metrics is used to measure and evaluate the handover processes homogeneously and heterogeneously within WiMAX environment. Three parameters have been selected for the evaluations which are Throughput, E-2-E and Jitter.

\subsubsection{Throughput}

The average Throughput represented by: the ratio of total amount of data that reaches its destination to the time taken for the data to transfer from the source to the destination. The data packets received at the physical layer are sent to the higher layers if they are destined for this station. Throughput is usually described by bytes or bits per second (Bps or bps).

$$
\text { Throughput }=\frac{\text { Total bytes received } * 8[\text { bit }]}{\text { End time }[s]-\text { Start time }[s]}
$$




\subsubsection{End-to-End (E-2-E) Delay}

The average End-to-End (E-2-E) Delay is the time taken for a packet to be transmitted across a network from source to destination. This metric describes the packet delivery time whereas the lower the E-2-E Delay, the better the application performance. The E-2-E Delay value is averaged over the number of packets. It is mathematically represented by the following equation.

$$
\text { Average } E-2-E \text { delay }=\frac{\text { Total } E-2-E \text { delay }}{\text { No.of packets received }}
$$

\subsubsection{Jitter}

The Jitter is the deviation in the time among packets arriving at the destination side, caused by network congestion, timing drift, or route changes. It signifies the Packets from the source till they reach their destination with different delays. A packet's delays vary with its location in the queues of the routers along the path between source and destination. This location is not predictable due to network circumstances. Equation below describes the Jitter calculation:

$$
\text { Jitter }=\text { Receptiom Time of packet(I)-Reception Time of packet(I-1) }
$$

\subsection{Simulation Parameters}

Various parameters have been used in all simulation scenarios to analyze the handover behavior under specific circumstances both homogeneously and heterogeneously. The simulation parameters that are used in this research are listed in Table 1. The speed of mobile nodes during handover process is $80 \mathrm{KMPH}$; the size of the field is $1500 \mathrm{~m} \mathrm{x} 1500 \mathrm{~m}$. During the simulation, all nodes start performing handover from 0 second until the time of simulation ends $(25 \mathrm{~s})$.

\subsection{Simulation Environments}

Table 1. Simulation parameters

\begin{tabular}{lc}
\hline & Simulation Parameters \\
Parameter & Value \\
\hline BS range radius $(\boldsymbol{m})$ & 1000 \\
AP range radius $(\boldsymbol{m})$ & 500 \\
Terrain-dimensions $(\boldsymbol{m})$ & 1500,1500 \\
BS-AP distance $(\boldsymbol{m})$ & 1200 \\
Frequency band $(\boldsymbol{G H Z})$ & 2.4 \\
Handover RSS trigger & -78.0 \\
Handover RSS margin & 3.0 \\
Channel bandwidth $(\boldsymbol{M H Z})$ & 20 \\
Frame duration $(\boldsymbol{m} \boldsymbol{s})$ & 20 \\
FFT size & 2048 \\
MS velocity $\left[\boldsymbol{m} . \boldsymbol{s}^{-1}\right]$ & 20 \\
BS transmit power $P_{t}=\frac{d B m}{h e i g h t}(m)$ & $20 / 5$ \\
AP transmit power $P_{t}=\frac{d B m}{h e i g h t}(m)$ & $20 / 1.5$ \\
MS transmit power $P_{t}=\frac{d B m}{\text { height }}(m)$ & $15 / 1.5$ \\
Somulation time $(\mathrm{s})$ & 25 \\
Traffic & $\mathrm{CBR}$ \\
\hline & \\
&
\end{tabular}

The QualNet simulator v.4.5.1 has been used to simulate the proposed scenarios for studying the handover process between WiMAX, WiFi and UMTS networks, as shown in Figure 4. UMTS and WiFi are chosen as they are common networks used nowadays and their technologies are almost similar. Therefore, it's possible to establish new connections between them heterogeneously.

The simulation of the handover process between the mentioned homogeneous and heterogeneous networks is conducted to evaluate the Throughput, E-2-E Delay and jitter. In each simulation scenario, the UCD and DCD messages are modified within two time intervals (5 and 10 seconds) during handover to find out which time interval performs better in each performance metric. The Constant Bit Rate (CBR) application is used to simulate the assumed scenarios in QualNet v.4.5.1. A snapshot for simulation scenario is shown in Figure 5.

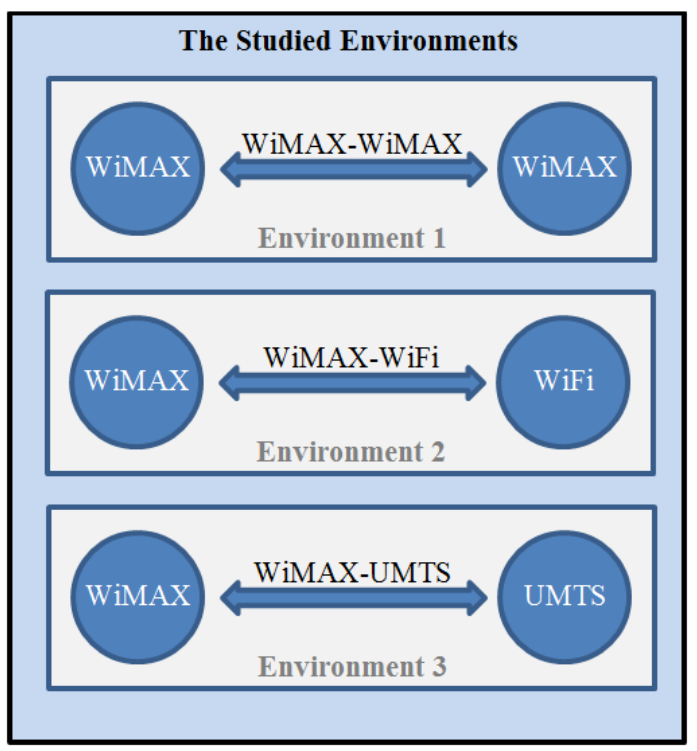

Figure 4. Simulated environments

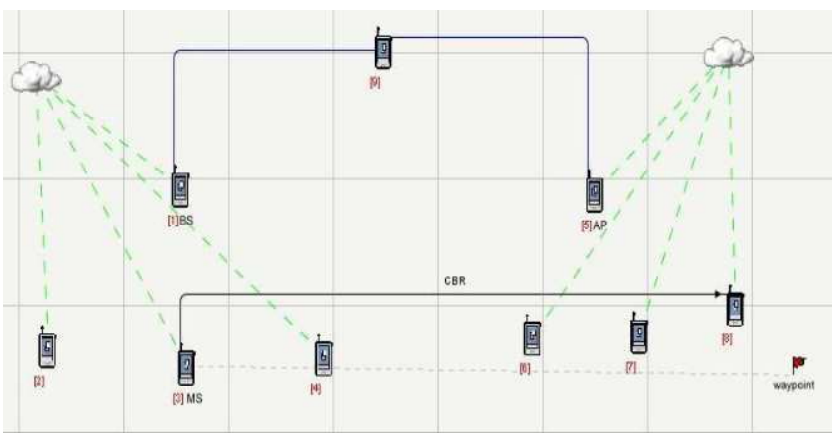

Figure 5. The QualNet simulation scenario of WiMAX and WiFi networks

The left side of Figure 5 illustrates the WiMAX network, the BS and three MSs associated to the BS. The other side, a WiFi network is configured with one access point (AP) node number 5, and three client nodes under the AP coverage.

The simulation setup corresponds to a connection of the WiMAX BS to another UMTS BS or WiFi AP (another network) by backhaul links T1 through a central gateway BS 
(node number 9). The MS (node number 3 ) is a mobile node that moves from its location on the waypoint and stops in the WiFi cell (for example of one moving node). However, the MS performs the handover process from WiMAX to WiFi coverage, while it is communicating or transmitting data to the destination node (node number 8) under WiFi access point (AP 5). In the meantime, the QualNet simulator collects the statistics for all performance metrics.

Four different experiments are conducted to evaluate the WiMAX handover mechanism. The first experiment is conducted to evaluate the average Throughput of the system during handover process. The second experiment compares the difference in the inter packet arrival times at the receiver (jitter) with respect to the total number of MS for every routing protocol. The purpose of the third experiment is to analyze the E-2-E Delay of the system during handover process. Finally, the fourth experiment is about comparing the time intervals of the UCD and DCD messages during handover process within two time intervals (5 and 10 seconds), regarding to the three performance metrics just mentioned above to find out which time interval performs the best during the handover process.

\section{Results}

Results obtained from the handover simulation process are discussed in this section. The Throughput, E-2-E Delay and Jitter of each simulated environment of the three (WiMAX-WiMAX, WiMAX-WiFi and WiMAX-UMTS) is discussed in the following sup-sections:

\subsection{Throughput Result}

Average Throughput is the ratio of total amount of data that reaches its destination to the time taken for the data to travel from the source to the destination. Figure 6 shows the average Throughput of the three handover environment with respect to the number of moving nodes.

The average Throughput in WiMAX-WiMAX handover is obviously the highest since it involves the same technology. In another words, the similarity in the BS type is high which saves any extra management signaling. WiMAX-UMTS comes in second, with slightly lower Throughput compared to WiMAX-WiMAX. WiMAX-WiFi comes in last, with much lower Throughput compared to WiMAX-WiMAX and WiMAX-UMTS. It is also observed that as the number of moving nodes increases, the average Throughput slightly decreases. Figure 6 depicts that the WiMAX-WiFi has much lower Throughput compared to the other two as a result of lower performance of the WiFi network compared with WiMAX and UMTS. It is also recorded that the Throughput drops dramatically with more moving nodes due to the high load on the network with more nodes.

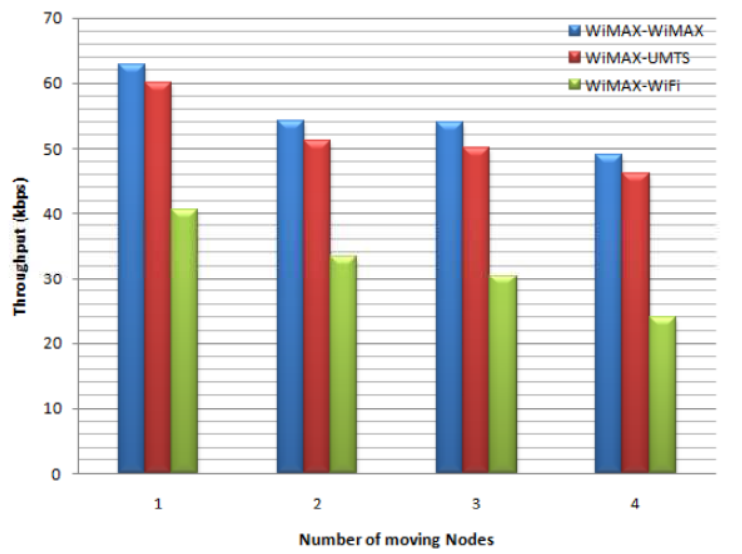

Figure 6. Overall Throughput for various number of moving nodes

Figure 7 below illustrates the system Throughput of WiMAX-WiMAX environment with variant values of UCD and DCD messages. Two time interval (5 and 10s) is configured in each simulation run. In first run, the time interval of UCD and DCD messages is 5 seconds for each. In second run, the time interval is 10 seconds for each.

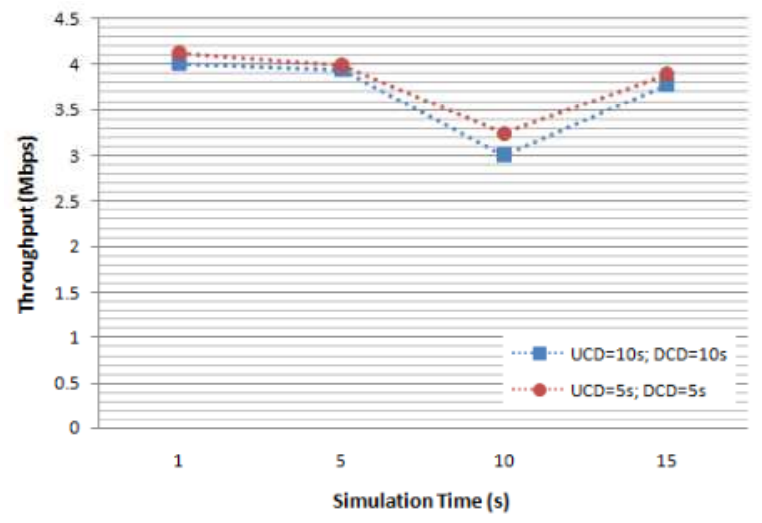

Figure 7. System Throughput of WiMAX-WiMAX environment with variant values of UCD and DCD messages

Consequently, the first case (with $5 \mathrm{~s}$ ) achieved higher Throughput than that of second case (with 10s). In fact, reducing acquisition time of such management messages (UCD and DCD) helps to enhance the network performance, thus, reducing the signaling time between BSs and MSs. Also, the MSs can be updated, by the BS, about the network information in shortest time (every $5 \mathrm{~s}$ ) which shows better performance, resulting here with higher Throughput. Figure 7 also shows that the performance of both cases is degraded in the simulation time $10 \mathrm{~s}$ (X-axis); this is due to the occurrence of handover process at this time and conversely, the system Throughput is dramatically decreased.

\subsection{End-to-End Delay Result}

In general, the average of E-2-E Delay matrix for all simulation environments (WiMAX-WiMAX, WiMAX-UMTS and WiMAX-WiFi) increases as the number of MSs in the network increases (see Figure 8). This is expected in wireless environments due to queuing delays 
at every hop node (BS or AP). Neither the WiMAX-WiMAX nor the WiMAX-UMTS utilize their effects on average E-2-E Delay for traffic; hence it appears to be minimal. However, the average E-2-E Delay for the different numbers of MSs with the WiMAX-WiMAX and WiMAX-UMTS are lower than with the WiMAX-WiFi under all scenarios. Similar to the result in Section A, the WiMAX-WiMAX and WiMAX-UMTS handover give the best performance while the WiMAX-WiFi gives the worst performance due to WiFi network lower performance compared with WiMAX and UMTS in addition to the variety in the technology specifications quality.

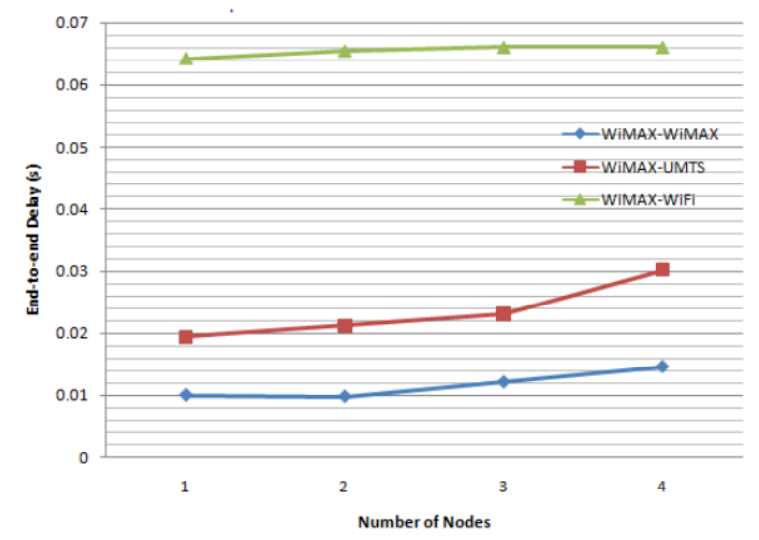

Figure 8. The average E-2-E Delay for the three simulated environments

Figure 9 below shows the average E-2-E Delay of WiMAX-WiMAX environment with variant time intervals of UCD and DCD messages. The first simulation run with $5 \mathrm{~s}$ achieves lower E-2-E Delay compared to the second run with 10s. This is due to minimizing the required time for managing messages signaling.

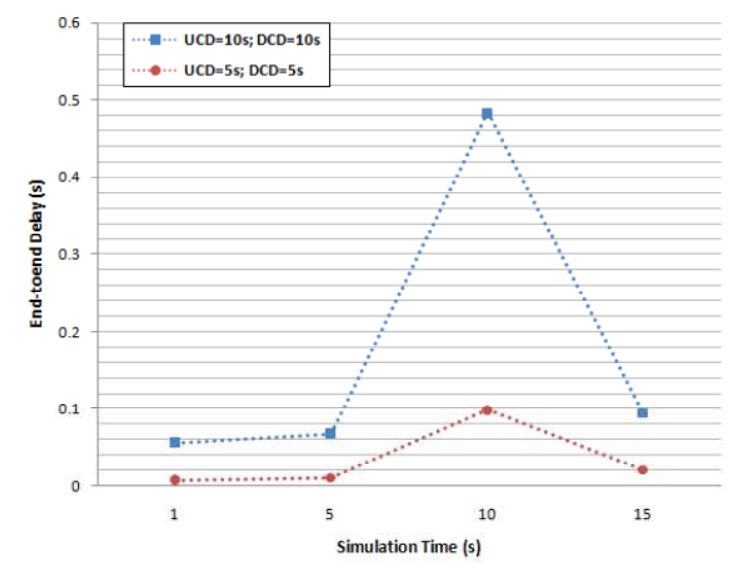

Figure 9. The average E-2-E Delay of WiMAX-WiMAX scenario with variant time intervals of $U C D$ and $D C D$ messages

In addition, the gap between both simulations run at the 10 s (i.e. the moment of the handover process) is much wider compared to other simulation times (e.g. 1, 5, and 15s). This confirms that the $5 \mathrm{~s}$ of UCD and DCD messages shows better performance compared to the $10 \mathrm{~s}$ time interval.

\subsection{Jitter Result}

The simulation result of the overall mobility Jitter performance with various number of moving MSs for all simulated environments is shown in Figure 10. The WiMAX-WiMAX and WiMAX-UMTS environments perform with lower Jitter compared with WiMAX-WiFi environment due to the variance in the technology specifications quality.

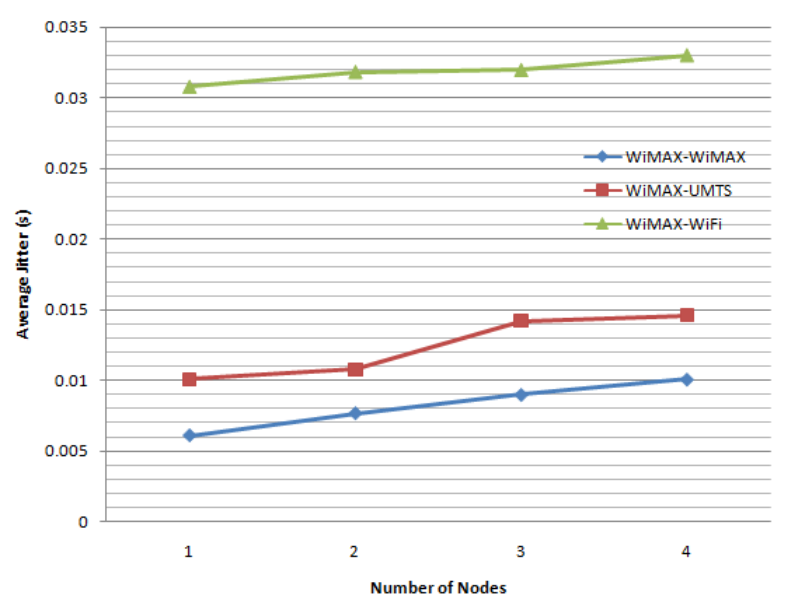

Figure 10. The overall mobility Jitter for versus number of moving MSs

The system with WiMAX-WiMAX environment in the first run of UCD and DCD messages with 5s time interval achieved lower Jitter compared to second run of 10s time interval as shown in Figure 11. This is due to the short time interval required for managing messages which reduces the time required for managing the messages signaling between nodes. More so, the first run with $5 \mathrm{~s}$, the simulation time at "10s" period shows better performance than the second run with 10 s time interval.

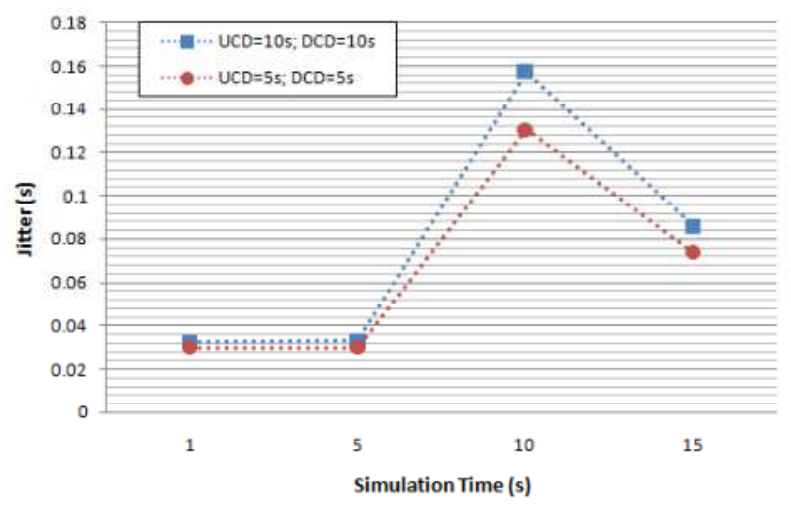

Figure 11. The system Jitter of the simulation scenario with WiMAX-WiMAX environment

\section{Discussion}

This paper presents an attempt to study and analyze the mobility in a collection of homogeneous and heterogeneous WiMAX, UMTS and WiFi environments regarding the handover process between such wireless networks. The 
main focus is to investigate the handover process in Mobile WiMAX in terms of its rules and address management and the main issues related to it. There is a limited related works that investigate the handover scenarios in networks' simulation analysis. They concentrate more on the transportation of subscribers (Mobile Stations) within WiMAX environment and with another environments (e.g. UMTS or WiFi), and suggest other fundamentals like scheduling and QoS as future works.

Two of the most important requirements for wireless communication technologies are to be applicable and universally desirable. Mobile WiMAX 802.16e handover mechanism analysis is the main target for this research. The focal point here is to introduce a complete understanding about handover process in WiMAX network, as it is considered as the most important process to achieve mobility within wireless networks. Work evaluation has been done by simulating handover process with different situations homogeneously and heterogeneously. The main task of the simulation process is to determine what parameters are affected during handover process in mobile networks homogeneously within WiMAX environment and heterogeneously to UMTS and WiFi networks.

The results show the ability to evaluate the performance of handover procedure within WiMAX and the other networks that have been chosen for this research. Based on the obtained results, a comparison among the three different networks has identified the best environment to establish a "handover".

Regarding to channel descriptor, the purpose of the DCD messages is to specify the characteristics of a given downlink physical channel. The BS transmits them at a given interval, which can have a maximum value of 10 seconds. It's the same for UCD, in that it specifies the characteristics of the uplink channel and is transmitted at a given interval, which cannot exceed 10 seconds.

The WiMAX-WiMAX case has been simulated using two time intervals ( 5 and 10 ) seconds under the three differential matrices (Throughput, E-2-E Delay and jitter) expecting that the time interval $5 \mathrm{~s}$ will achieve the best results due to the short time it takes to update the current information compared to $10 \mathrm{~s}$.

Best results have been obtained from the simulation of WiMAX-WiMAX followed by WiMAX-UMTS while WiMAX-WiFi shows the worst. WiMAX-WiMAX has achieved the highest amount of Throughput and the lowest E-2-E Delay and jitter. Furthermore, a comparison has been done to find out which time interval achieves the best results within the same network WiMAX-WiMAX. The time interval $5 \mathrm{~s}$ achieved the best outcomes comparing to the other time interval $10 \mathrm{~s}$ regarding to the three parameter metrics.

\section{Conclusion and Future Work}

We discuss the simulation results of the handover process over WiMAX-WiMAX, WiMAX-UMTS and
WiMAX-WiFi environments/networks. The simulation scenarios are simulated to compare the results of each environment's behavior with handover process using the QualNet simulator v4.5.1. The environment (WiMAX-WiMAX) has shown substantial enhancement of the system Throughput, reduction of E-2-E delay, and reduction of Jitter. This is mainly due to the similarity in the BS type which saves any extra management signaling. For example, between WiMAX BS and UMTS BS, the management messages signaling require longer time compared with WiMAX-WiMAX BSs.

In addition, the best environment (WiMAX-WiMAX) is simulated with various values of the time intervals of UCD and DCD management messages as a deep analysis. However, all related simulation results show that the shorter time interval of both UCD and DCD messages improves the overall network performance and the handover process.

Based on the analyses that have been done in this project, an optimization is required for the handover process of mobile WiMAX to improve its performance in terms of Delay and moving speed. Thus, the handover optimization in mobile WiMAX is the recommendation for future work, such as the proposed scheme that has been expressed in [24].

\section{Acknowledgements}

We would like to thank all the college of graduate studies staff of the Universiti Tenaga Nasional for their help and support during our study in the MIT program 2010/2012.

\section{References}

[1] IEEE 802.16 Working Group. "IEEE Standard for Local and Metropolitan Area Networks, Part 16: Air interface for fixed broadband wireless access systems," IEEE Std 802 (2004).

[2] L. M. Carlberg, and A Dammander, "WiMAX-A study of mobility and a MAC-layer implementation in GloMoSim," Master's Thesis in Computing Science (2006).

[3] D. H. Lee, K. Kyamakya and J. P. Umondi, "Fast handover algorithm for IEEE 802.16 e broadband wireless access system," Wireless pervasive computing, 2006 1st international Symposium on. IEEE, 2006.

[4] A. F. Kabir, M. Khan, R. Hayat, A. A. M. Haque and M. S. I. Mamun, "WiMAX or Wi-Fi: The Best Suited Candidate Technology for Building Wireless Access Infrastructure," arXiv preprint arXiv:1208.3769 (2012).

[5] M. F. Finneran, "WiMAX versus WiFi a comparison of technologies markets and busniss plan," dBrn associates Inc, 2004.

[6] A. Ezzouhairi, Q. Alejandro and S. Pierre, "Towards cross layer mobility support in metropolitan networks," Computer Communications , ELSEVIER 33.2 (2010): 202-221.

[7] A. S. Rashid, A. A. Hassan, M. Amitava, F. Francisco and W. K. Daniel, "WiMAX, LTE, and WiFi interworking," Journal of Computer Systems, Networks, and Communications, 2010. 
[8] Q. B. Mussabbir, "Mobility management across converged IP-based heterogeneous access networks," PhD Thesis. School of Engineering and Design, Brunel Universit (2010).

[9] International Telecommunication Union (ITU), "Broadband mobile communications towards a converged world," Micworkshop on shaping the future mobile information society, Seoul. 2004.

[10] I. F. Akyildiz, J. Xie and S. Mohanty. "A survey of mobility management in next-generation all-IP-based wireless systems," Wireless Communications, IEEE 11.4 (2004): $16-28$.

[11] Z. Becvar, P. Mach and R. Bestak, "Initialization of handover procedure in WiMAX networks," ICT-MobileSummit 2009 Conference Proceedings, 2009.

[12] J. Liao, Q. Qi, X. Zhu, Y. Cao, and T. Li, "Enhanced IMS handoff mechanism for QoS support over heterogeneous network," The Computer Journal 53.10 (2010): 1719-1737.

[13] K. R. Santhi and G. S. Kumaran, "Migration to 4 G: Mobile IP based solutions," Telecommunications, 2006. AICT-ICIW'06. International Conference on Internet and Web Applications and Services/Advanced International Conference on. IEEE, 2006.

[14] WiMAX.com, "What is WiMAX," WiMax.com Broadband $\begin{array}{lcc}\text { Solutions, } & \text { Inc. } & 2011 . \\ \text { http://www.wimax.com/general/what-is-wimax }>\end{array}$

[15] Chen, Kwang-Cheng, J. Roberto B. de Marca, and J. Roberto, eds. Mobile WiMAX. John Wiley, 2008.

[16] Z. Becvar and J. Zelenka, "Handovers in the mobile WiMAX," Research in Telecommunication Technology 1 (2006): 147-50.
[17] D. Pareit, B. Lannoo, I. Moerman and P. Demeester, "The history of WiMAX: A complete survey of the evolution in certification and standardization for IEEE 802.16 and WiMAX," Communications Surveys \& Tutorials, IEEE 14.4 (2012) 1183-1211.

[18] K. R. Sayan, R. S. Kumar, K. Pawlikowski, A. McInnes, H. Sirisena "A fast and simple scheme for mobile station-controlled handover in mobile WiMAX," Access Networks. Springer Berlin Heidelberg, 2011. 32-44.

[19] A. Shami, M. Maier, and C. Assi. Broadband Access Networks: Technologies and Deployments. Springer Verlag, 2009.

[20] F. Muratore. UMTS: Mobile communications for the future. John Wiley \& Sons, Inc., 2000.

[21] QualNet 4.5 Programmer's Guide, Scalable Network Technologies, Inc., $2008 . \quad<$ http://www.eurecom.fr/-chenj1QuaINet03.pdf.>

[22] QualNet Product Family, Scalable Network Technologies, Inc., $\quad 2010$. $<$ http://www.scalable-networks.com/pdf/QualNet_Family.pd f>

[23] Y. F. Dong, and R. Liu, "Simulation studies of reliable data delivery protocols," CSIRO ICT Centre, Australia 2007.

[24] W. Jiao, P. Jiang and Y. Ma, "Fast handover scheme for real-time applications in mobile WiMAX," Communications, 2007. ICC'07. IEEE International Conference on. IEEE, (2007): 6038-6042. 